\title{
High-Resolution Mass Spectrometry of Large Molecules in a Linear Time-of-Flight Mass Spectrometer
}

\author{
Gary R. Kinsel*, Johann M. Grundwuermer, and Juergen Grotemeyer \\ Institut fuer Physikalische und Theoretische Chemie der Technischen Universitaet Muenchen, Garching, \\ Germany
}

\begin{abstract}
This report describes a new high-resolution linear time-of-flight mass spectrometer that has been constructed at this institute. The instrument is used for investigations of both direct and matrix-assisted laser desorption/ionization of large molectles. A unique feature of this new instrument is the incorporation of a $10-\mathrm{cm}$ postsource pulse-focusing region for enhancing the resolution of the detected ion signals. This technique can correct for both high initial ion translational energies and long durations of ion formation and is expected to be particularly well suited for laser desorption/ionization applications. Results of calculations are presented to illustrate the gains in mass resolution that may be expected for a variety of ion formation conditions. In addition, initial experimental results are presented that demonstrate the capability of this new instrument to produce high-resolution ion signals. Signals with mass resolutions as high as $\mathbf{2 7 5 0}$ (full width at half maximum) have been obtained for both direct and matrix-assisted laser desorption/ionization signals. (T Am Soc Mass Spectrom 1993, 4, 2-10)
\end{abstract}

$\mathrm{T}$ The origin of direct laser desorption/ionization (DI.D/I) as a method for introducing ions from pure samples into the gas phase for mass spectral analysis may be traced to the early 1960 s [1-3]. Despite the general observation that DLD/I appears limited to analysis of molecules with masses less than a few thousand daltons, research and application continue to extend this method to higher masses, and a variety of reports may be found in the recent literature $[4,5]$. Recently, it has been shown that the addition of a matrix that absorbs strongly at the desorbing laser wavelength to the sample of interest allows the introduction of much larger ions into the gas phase $[6,7]$. This matrix-assisted laser desorption/ionization (MALD/l) technique has been used to successfully introduce ions with masses greater than 100,000 Da into the gas phase for subsequent mass spectral analysis [8]. This achievement has sparked renewed interest in the area of laser desorption/ionization (LD/I), and the abundance of reports in the past few years using MALD/I attest to the enormous promise of this new approach [9-13]. \footnotetext{
*Present address: Texas A \& M University, Department of Chemistry.
College Station, TX 77843.

Address reprint requests to Juergen Grotemeyer, Institut fuer Physikalische Chemie, Universitaet Wuerzburg, Marcusstr. 9/11, D8700 Wuerzburg, Germany.
}

Both DLD/I and MALD/I methods are commonly coupled with a time-of-flight mass spectrometer (TOFMS) for mass analysis of the resulting ions. The inherent compatibility of pulsed-ion formation methods with TOF mass analysis makes combining these techniques a logical option. Complete mass spectra may be recorded for every laser shot, with very high sensitivities and at very high repetition rates. The principal drawback of the TOF-MS is the limited resolution of the resulting ion signals. For DLD/I applications, mass resolutions of ca. 1000 [full width at half maximum (FWHM)] are commonly observed when desorbing ions of relatively high molecular weight [14]. For MALD/I applicalions, the original spectra showed mass resolutions of only ca. 50 (FWHM) using a reflectron time-of-flight mass spectrometer (RETOF-MS) [15]. Such low resolutions may be due to very large spreads in ion translational energies or other factors that cannot be corrected by the reflecting field. Beavis and Chait [16] demonstrated that the mass resolution could be improved to ca. 700 (FWHM) in a linear TOF-MS through careful control of sample preparation and desorbing laser power. More recently, mass resolutions as high as ca. 1000 (FWHM) have been achieved in a RETOF-MS through improvements in instrument design [17, 18]; however, there is still a need for higher mass resolutions to be obtained, particulary when investigating high-mass ion signals. Higher resolutions 
are required for improvements in mass calibration and for distinguishing among various protonated, sodiated, or adduct ions, or all three, that may be formed during LD/I.

Resolution of ion signals in a TOF-MS has been the subject of numerous reports since the initial demonstration of this instrument, and excellent reviews may be found in the literature [19]. Primarily, resolution is determined by three components of the ion formation conditions. These three components are the spatial width over which the ions are produced, the distribution of the initial ion velocities, and the time duration over which the ions are formed [20]. Of these three components, the spatial width over which the ions are formed is generally assumed to be very small for LD/I experiments. A number of efforts have been made to measure the distribution of initial ion kinetic energies in LD/I [21]. For DLD/I, values of as much as several electron volts have been measured [22]. Recent reports on MALD / $I$ have also found kinetic energies ranging from a few to several tens of electron volts, and it has been suggested that the kinetic energy of the desorbed sample ions appears to increase as the size of the sample molecule increases [23]. The time component of ion formation in LD/I experiments is an ill-defined parameter, although it is often assumed that ions are only formed during the length of the desorbing laser pulse. This assumption has not been experimentally verified, however, and it has been suggested that one reason for the low-resolution ion signals observed in MALD/I experiments using a RETOF-MS may be that the time for ion formation is longer than that for the desorbing laser pulse [24].

Over the years, a number of theoretical studies of the factors determining mass resolution in a TOF-MS have been performed that have led to a variety of suggested approaches for enhancing the mass resolution. Perhaps the most successful of these approaches has been the RETOF-MS, which has the ability to correct for either large initial ion velocity or spatial distributions [25-27]. Resolutions of more than 20,000 (FWHM) have been achieved using this instrument design in combination with short ionizing laser pulses or supersonic jet sample introduction, or both; however, it should be recognized that no static field TOFMS, including a RETOF-MS, is capable of correcting for long ion formation times. Ihis problem can, however, be solved by using a time-dependent acceleration of the ions. Several time-dependent ion acceleration approaches have been suggested in the literature, including time-lag focusing [28], velocity compaction [29], impulse field focusing [30], dynamic field focusing [31], and postsource pulse focusing (PSPF) [32, 33], each of which has the potential capacity to correct for distributions in the duration of ion formation.

Of these techniques, PSPF presents a number of attractive features that may be considered. First, the simplicity of PSPF makes implementation of the technique relatively straightforward. In PSPF the ions are accelerated from the TOF-MS source region by means of conventional two-stage static electric fields and subsequently enter a short, initially field-free pulse-focusing region. After the ions have entered this region, a voltage pulse is applied to the region that compresses the individual mass ion packets, yielding higher resolution ion signals at the detector. Implementation of PSPF therefore requires simply the addition of a short region adjacent to the ion source region, a pulsed-voltage supply, and a time-delay generator to offset the voltage pulse from the ionizing laser pulse. Although such focusing cannot cover the entire mass spectrum, a relatively large fraction of the mass spectrum may be focused without serious sacrifices in ion collection or resolution for the remainder of the mass spectrum. Depending on the length of the pulse-focusing region and the ion-formation parameters, the fraction of the mass spectrum focused may be $80 \%$ or more relative to the highest mass ion focused using conventional static instrumental conditions.

We have constructed a 2-m linear TOF-MS that incorporates a $10-\mathrm{cm}$ PSPF region adjacent to the ion source region. This instrument will be used for investigations of both DLD/I and MALD/I. We present here a detailed description of the instrumental construction, some theoretically predicted operational characteristics, and initial results of application of PSPF to both DLD/I and MALD/I ion signals. Both the calculational predictions and the initial experimental results illustrate the significant resolution enhancement that can be achieved with PSPF.

\section{Experimental}

In Figure 1 a schematic of the instrumental arrange ment is presented. The basic TOF-MS structure consists of a cylindrically bored, cubic aluminum source region chamber attached to a $2-\mathrm{m}$ stainless steel flight tube. The source chamber may be isolated from the flight tube by using a 6-in. gate valve (VAT, Grafing, Germany) installed between the two components. The source chamber is pumped by a $330-\mathrm{L} / \mathrm{s}$ turbomolecular pump (Balzers, Wiesbaden, Germany), and the flight tube is pumped by a second $150-\mathrm{L} / \mathrm{s}$ turbomolecular pump (Leybold-Heraus). Typically, an operating vacuum of ca. $1 \times 10^{-6}$ torr, as measured by two ionization gauges mounted separately in the source chamber and flight tube (Balzers TPG 300, IKR 020), is maintained using this pumping system.

Figure 2 shows the ion source and acceleration region assembly in expanded detail. The acceleration region assembly consists of a series of 13 stainless steel acceleration plates, each separated by four Teflon spacers, mounted on a horizontally and vertically micrometer-adjustable flat. Each of the acceleration plates measures $52.0 \times 52.0 \times 1.0 \mathrm{~mm}$. Plates 1 and 2 are separated by $10.0 \mathrm{~mm}$ and define the ion source region. Plates 2 and 3 are separated by $5.0 \mathrm{~mm}$ and define the secondary acceleration region. Plates 3-13 


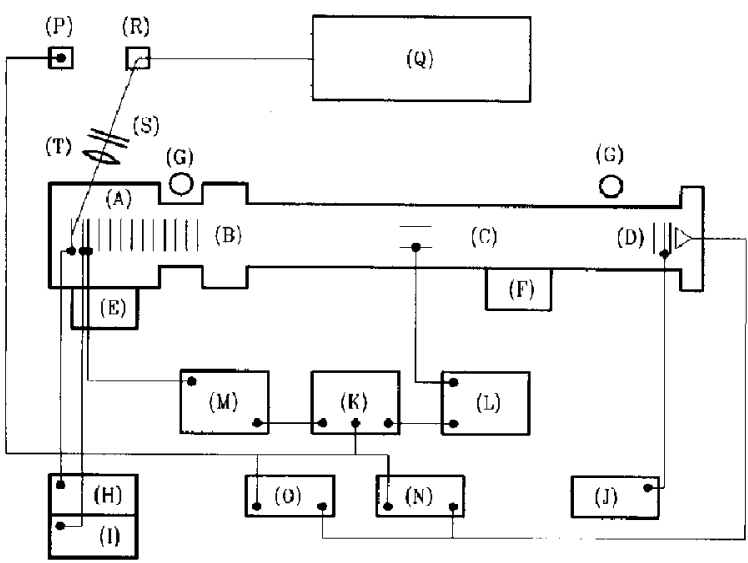

Figure 1. Block diagram of the 2-m linear TOF-MS instrument (see text for a detailed description of the various components): (A) source chamber; (B) gate valve; (C) $2-\mathrm{m}$ flight tube; (D) dual microchannel plate detector; (E) 330-L/s turbomolecular pump; (F) 150-L/s turbomolecular pump; (G) vacuum ionization gauges; (H) acceleration plate 1 power supply; (I) acceleration plate 2 power supply; (J) detector power supply; (K) digital delay generator: (L) pulsed-voltage supply for ion ejection plates; (M) pulsed-voltage supply for pulse-focusing region; (N) $350-\mathrm{MHz}$ transient oscilloscope; (O) 200-MHz transient digitizer; (P) triggering fast photodiode; (Q) Nd:YAC laser; (R) Pellin-Broca beam separator; (S) neutral density filters; (T) $15-\mathrm{cm}$ quartz cylindrical focusing lens.

are each separated by $9.0 \mathrm{~mm}$ and define the pulsefocusing region. From plate 13, which defines the end of the pulse-focusing region, to the grounded grid in front of the ion detector measures $1899.0 \mathrm{~mm}$. The plates in the pulse-focusing region are connected via a series of $10.1-\mathrm{k} \Omega$ resistors. Acceleration plates 2-13 each have a $10.0-\mathrm{mm}$ hole bored through their centers for transmission of the ions. Nickel, $90 \%$, transmission grids (Buckbee-Mears, Muenchen, Germany) are fixed with conducting glue over the holes in acceleration plates 2,3 , and 13 to define the initial and postacceleration electric fields.

Samples may be introduced into the source cham-

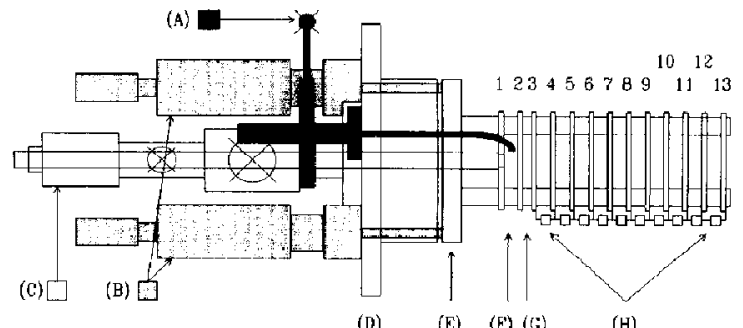

(D) (E) (F) (G)

(H)

Figure 2. Expanded details of the ion source and acceleration region assembly (see text for detailed description of the various components): (A) molecular leak inlet; (B) $X Y$ micrometer deflectors; (C) direct insertion probe; (D) vacuum flange; (E) micrometer-adjustable flat on which the acceleration assembly is constructed; (F) ion source region; (G) secondary acceleration region; (H) pulse-focusing region. Numbers 1-13 refer to the 13 acceleration plates and are described in the text. ber by either of two methods. A molecular leak inlet connected to a needle tube in the vacuum chamber may be used for introducing volatile sample species. The needle serves to direct the sample vapor to the ion source region. For nonvolatile samples, a direct insertion probe is available that enters the source chamber from behind the acceleration region assembly. The spring-mounted, polished stainless steel probe tip enters the ion source region through an $8.0-\mathrm{mm}$ hole bored in acceleration plate 1 . When ionizing gas phase samples, the uncoated probe is placed in position to fill the hole in acceleration plate 1 and better define the source region electric field.

The electronic components consist of five power supplies (FUG Elektronic, Rosenheim, Germany), a digital delay generator (Stanford Research Systems, DG 535, SI Instruments, Gilching, Germany), and two in-house-constructed pulsed-voltage supplies. Two power supplies with maximum outputs of $12.5 \mathrm{kV}$ are used for static voltage biasing of the first and second acceleration plates. A third power supply is used for biasing of a dual microchannel plate detector with a total postacceleration of $-2.0 \mathrm{kV}$. The fourth and fifth $1.25-\mathrm{kV}$ power supplies are used to power the two pulsed-voltage generators. These pulsed-voltage generators are designed to produce a high-voltage pulse that follows the waveform of a TTL pulse produced externally by the digital delay generator. They are capable of delivering voltage pulses of up to $1.25 \mathrm{kV}$ for durations of up to $40 \mu \mathrm{s}$ with rise times of ca. 40 ns. One pulsed-voltage supply is used to drive a pair of ion ejection plates positioned $1.0 \mathrm{~m}$ away from the ion source region in the mass spectrometer flight tube. The ion ejection plates may be used to eject low-mass matrix ions produced during MALD/I expcriments. The second pulsed-voltage supply is used to deliver a voltage pulse to acceleration plates $3-13$ for pulse focusing of the detected ion signals. In practice the voltage pulse is delivered to acceleration plate 3 , whereas acceleration plate 13 is maintained at ground, generating a constant accelerating electric field across the pulse-focusing region. The timing of both voltage pulses may be controlled independently by using the digital delay generator.

Ion detection is accomplished by using a standard dual microchannel plate detector positioned at the end of the 2-m flight tube. The distance from the grounded grid defining the end of the field-free flight tube to the detector surface is $15.0 \mathrm{~mm}$. The transient current from the ion signals is terminated through a $50 \mathrm{l}$ impedance collector and fed to a $350-\mathrm{MHz}$ oscilloscope (Tektronix 2465A CT, Tektronix Inc., Beaverton, OR) for continuous monitoring of the ion signals. This signal may be further transferred to an in-house-constructed $200-\mathrm{MHz}$ transient digitizer for spectra collection and storage. The digital delay generator, $350-\mathrm{MHz}$ oscilloscope, and $200-\mathrm{MHz}$ transient digitizer are all triggered by using the output of a fast photodiode positioned to detect a reflection of the desorbing laser pulse. 
Desorbing laser radiation is provided by the output of a Nd:YAG laser (QuantaRay, DCR 11-A, Spectra Physics, Darmstadt, Germany) passed through the appropriate doubling crystals to produce either the third harmonic at $355 \mathrm{~nm}$ or the fourth harmonic at $266 \mathrm{~nm}$. The output laser radiation is turned $90^{\circ}$ through a Pellin-Broca prism that acts to separate the various fundamental and harmonic wavelengths. The desired wavelength is selected by using appropriate beam stops and then passed through a series of neutral density filters to produce power densities of the desired magnitude. The laser beam is then focused to a spot size of ca. $0.0005 \mathrm{~cm}^{2}$ onto the sample-containing surface of the direct insertion probe at an angle of $25^{\circ}$ to the surface plane by using a $15-\mathrm{cm}$ cylindrical lens. The Nd:YAG laser may be operated either in the single-shot mode or at a repetition rate of $10 \mathrm{~Hz}$. Each laser shot produces a plume of desorbed ionic and neutral material that may contain both intact and fragmented molecules of the sample material. The ions produced in this way are accelerated and detected in the linear TOF-MS by using the components described.

\section{Calculational Results}

A thorough discussion of the theoretical basis of PSPF has been presented in the literature as well as a variety of simulations of expected mass resolution for gasphase ionization applications [32]. Numerous theoretical simulations were also performed before beginning construction of the new linear TOF-MS described in this report. Simulations were primarily directed at determining desired operational parameters and expected mass resolutions under a variety of ion formation conditions that might be anticipated during $L D / 1$ experiments. These simulations are based on ideal ion formation and detection conditions and do not take into account such effects as electric field distortions, detector response functions, and jitter in detection electronics; however, the calculated results and the accompanying discussion serve to illustrate the range of expected performance characteristics for the linear TOF-MS design described as well as a number of important points to consider when comparing PSPF and static electric field TOF-MS instrumentation.

The calculational method used to perform the simulations has been described in detail elsewhere [32]. Briefly, once the instrumental parameters, including region lengths, accelerating voltages, and timing for the focusing voltage pulse, have been defined, flight times for a group of ions of a given mass are calculated from first principles. These ions are chosen to be representative of the various distributions in initial ion velocities, points of formation, and formation times. Flight times are calculated for all representative ions, and the difference in detection time between the slowest and fastest ions determines the full width at the base (FWB) of the detected ion signal. The appropriateness of the representative ions chosen may be con- firmed by expanding the representational set and repeating the simulation until no change in calculated resolution is obtained. Mass resolution of the ion signal is then calculated by dividing the average flight time of the ion signal by the base width (f.t./FWB) and yields a value for which $50 \%$ valley resolution may be expected at that mass. By changing the choices used for the initial ion formation parameters, optimal operating conditions and expected mass resolutions over a wide variety of initial ion formation conditions may be determined.

Figure 3 shows two graphs of the mass resolution theoretically predicted for an ion with $m / z 1000$ under both static electric field and PSPF conditions over a range of initial ion translational temperatures and for three different total durations of ion formation, $d t$. The spatial distribution over which the ions are created is taken to be zero for these LD/I simulations. The ion translational temperatures given in the plots define the standard deviation $(k T / m)^{1 / 2}$ of the one-dimensional Gaussian distribution of initial ion velocities. Only ion velocity values ranging from 0 to +3 times the standard deviation are simulated, because desorption from
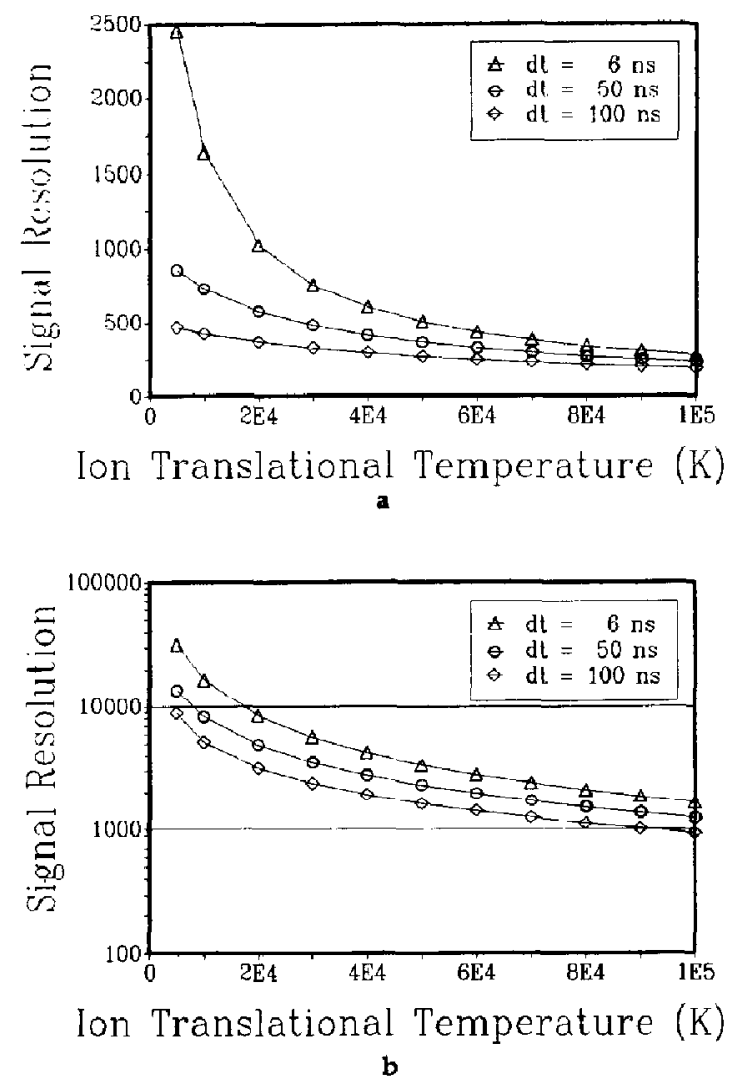

Figure 3. Plots of mass resolution (f.t. $/$ FWB) at $m / z 1000$ as a function of ion translational temperature for three different durations of ion formation, $d t$, under (a) static acceleration electric field conditions and (b) PSPF conditions. Instrumental configuration and acceleration voltages are described in the text. 
a surface prevents ions from being formed with initial velocities away from the flight tube. Therefore, a translational temperature of $50,000 \mathrm{~K}$ would correspond to a group of ions having a distribution in kinetic energy ranging from 0.0 to $6.5 \mathrm{eV}$.

For the static electric field simulations, it is assumed that acceleration plate 1 is biased at $7000 \mathrm{~V}$ and that all other acceleration plates are maintained at ground, yielding a $7000-\mathrm{V} / \mathrm{cm}$ electric field in the ion source region. For the PSPF simulations, it is assumed that acceleration plate 1 is at $7000 \mathrm{~V}$ and acceleration plate 2 is at $6500 \mathrm{~V}$ and that a focusing voltage pulse of between 850 and $1050 \mathrm{~V}$ is applied to acceleration plate 3 after the $m / z 1000$ ions enter the pulse-focusing region. All other parameters used in the simulations reflect the instrumental configuration described.

In the static electric field simulations (Figure 3a), it can be seen that at the lowest ion translational temperatures and shortest durations of ion formation, $d t$, a resolution of 2500 should be achievable. Once either the ion translational temperature or duration of ion formation begins to increase, the resolution falls off dramatically such that resolutions of only a few hundred are possible at the larger values simulated. The PSPF simulations (Figure 3b) clearly illustrate the potential gains of this approach. At low ion translational temperatures, resolutions of 9000 or greater are possible, even if the ions are produced for a time duration $d t=100 \mathrm{~ns}$. The actual base width of the ion signals in these simulations is much less than the 100-ns ion formation duration and indicate that significant focusing of the initial time component of ion formation may be achieved. As the ion translational temperatures increase, mass resolution also decreases when using the PSPF method; however, in all cases the predicted PSPF mass resolution is significantly better than that expected for static electric field acceleration.

It may be argued, however, that the comparison in Figure 3 is biased owing to the restriction of an acceleration electric field of just $7000 \mathrm{~V} / \mathrm{cm}$ in the static electric field simulations. Most current LD/I linear TOF-MS instrumentation uses very large initial acceleration electric fields to improve resolution of the detected ion signals. In Figure 4 the acceleration plate 1 voltage restriction is removed, and this voltage is regressed until maximum mass resolution is obtained over the same range of initial ion translationtal temperatures, with $d t=6 \mathrm{~ns}$. It can be seen that even after optimizing the voltage on acceleration plate 1 for maximum mass resolution, the resolution obtained is never as good as that obtained in the PSPF simulations. In addition, the acceleration plate 1 voltage required to produce these maximum mass resolutions climbs to over $100,000 \mathrm{~V}$ at the higher ion translation temperatures (Figure $4 b$ ).

Simulations also show that the use of higher initial acceleration voltages in combination with larger focusing voltage pulses may also be used to improve the mass resolution obtained with PSPF. In Figure 5a the
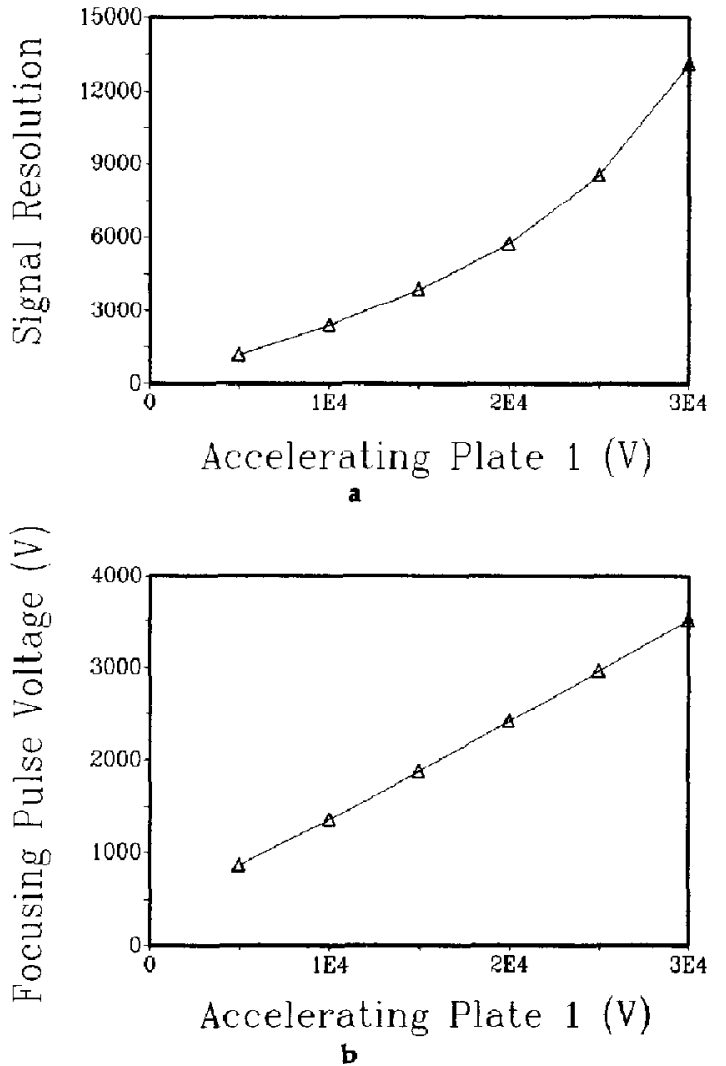

Figure 4. (a) Maximum mass resolution (f.t. $/ F W B$ ) at $m / z 1000$ and $d t=6$ ns for static acceleration electric field conditions obtained by optimizing the voltage applied to acceleration plate 1. (b) Optimum acceleration plate 1 voltage necessary to produce the mass resolution shown in a. All other instrumental configuration conditions are the same as in Figure 3 and are described in the text.

$m / z 1000$ mass resolution as a function of voltage applied to acceleration plate 1 is shown. In these simulations it is assumed that the ions have an initial translational temperature of $100,000 \mathrm{~K}$ and that $d t=6$ $n s$. In addition, the electric field in the first acceleration region is always held constant at $500 \mathrm{~V} / \mathrm{cm}$ (i.e., the acceleration plate 2 voltage is always $500 \mathrm{~V}$ less than the acceleration plate 1 voltage). Figure $5 \mathrm{~b}$ shows the magnitude of the focusing voltage pulse required to produce the maximum mass resolution shown in Figure $5 \mathrm{a}$. Note that the ion formation parameters used to generate Figure 5 are equivalent to the severest case simulated for the static electric field conditions shown in Figure 4, where the maximum mass resolution achievable for these ion formation parameters is just 1300 and requires an acceleration plate 1 voltage of over $130,000 \mathrm{~V}$. From Figure 5 it is clear that dramatic improvements in mass resolution over these values may be achieved by using PSPF. Mass resolutions of over 10,000 may be achieved at an acceleration plate 1 voltage of $30,000 \mathrm{~V}$ in combination with a $3500-\mathrm{V}$ 

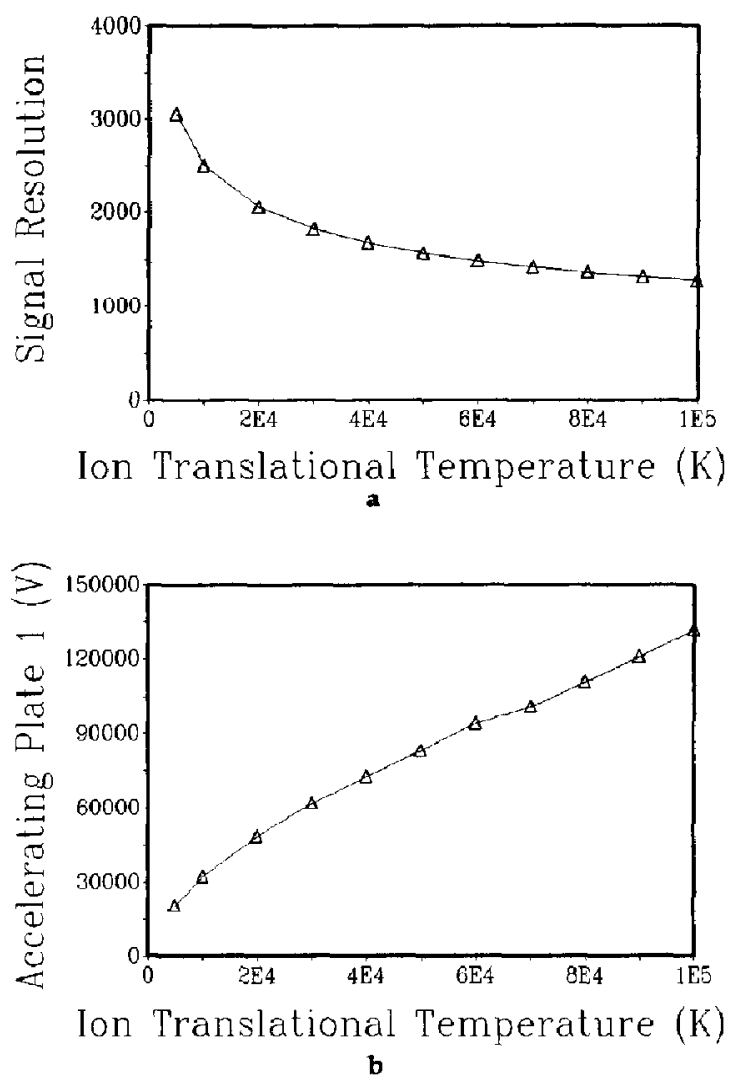

Figure 5. (a) PSPF mass resolution (f.t. $/ F W B$ ) at $m / z 1000$ for $d t=6 \mathrm{~ns}$ and an ion translational temperature of $100,000 \mathrm{~K}$ as a function of increasing acceleration plate 1 voltage. In these simulations, the acceleration plate 2 voltage is always defined as 500 $V$ less than the acceleration plate 1 voltage. (b) The focusing voltage pulse necessary to produce the mass resolution shown in a. All other instrumental configuration conditions are the same as in Figure 3 and are described in the text.

focusing pulse. These results are achieved despite the high ion translational temperature of $100,000 \mathrm{~K}$ and suggest that PSPF will be superior to very high static electric fields for producing high-resolution ion signals in linear TOF-MS instrumentation.

\section{Initial Experimental Results}

The experimental procedure used here is similar to that used with samples of high volatility [33]. At the start, the desired acceleration electric voltages are established, and an LD/I ion signal is obtained without the focusing voltage pulse. Next, the focusing voltage pulse is applied to the pulse-focusing region but timed to occur after the ions to be focused have entered the flight tube. An equivalent mass spectrum to the spectrum produced without the focusing voltage pulse is observed. The delay time for the focusing voltage pulse is then gradually reduced to shorter times until the ions being focused are within the pulse-focusing region at the time of the voltage pulse. A shift in the flight time of the ions to somewhat shorter values is observed owing to the additional acceleration of the voltage pulse. The delay for the focusing voltage pulse is further reduced until maximum mass resolution is observed. Finally, the mass resolution is further optimized through fine adjustment of the magnitude of the focusing voltage pulse.

This relatively simple procedure was applied to a number of medium molecular weight (MW) ion signals produced using both DLD/I and MALD/I to test the newly constructed linear TOF-MS and to demonstrate the effectiveness of the PSPF method. Figure 6 shows DLD/I spectra of zinc mesoporphyrin (MW 656 Da) both with and without PSPF. The 266-nm laser desorbing power was held constant at ca. $10 \mathrm{MW} / \mathrm{cm}^{2}$ for both spectra. The sample was prepared by dissolving zinc mesoporphyrin in a solution of methylene chloride, aspirating the solution through a syringe nee-
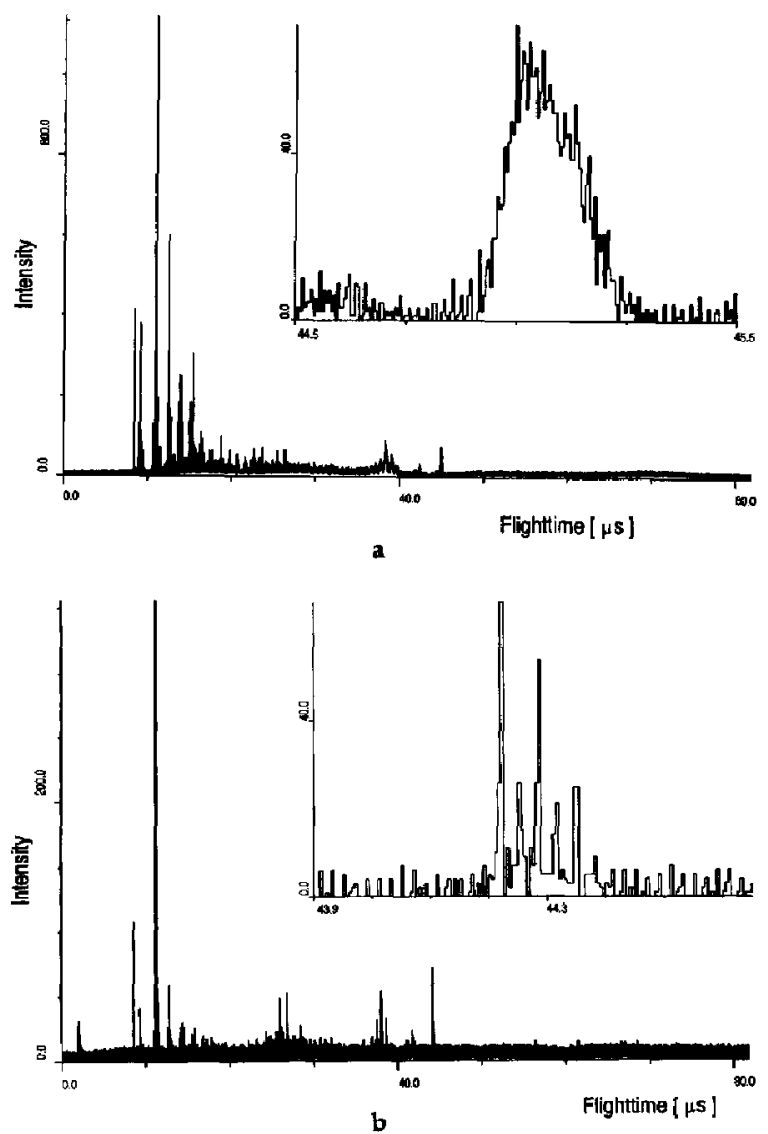

Figure 6. (a) DLD/I signal of zinc mesoporphyrin (MW $656 \mathrm{Da}$ ) taken under static accelerating electric field conditions; (b) DLD/I signal of zinc mesoporphyrin taken with PSPF to enhance the resolution of the molecular ion. Insets show expansions of the molecular ion regions. Both spectra represent the sum of five laser shots. 
dle, and then air-spraying the solution in the form of small droplets onto the stainless steel probe tip. For the unfocused mass spectrum (Figure 6a), the static source region accelerating electric field was set at $7000 \mathrm{~V} / \mathrm{cm}$ by biasing acceleration plate 1 at $7000 \mathrm{~V}$ and acceleration plate 2 at $0 \mathrm{~V}$. The resolution of the molecular ion signal under these conditions was just 120 (f.t./FWB). To use PSPF, acceleration plate 1 was biased at $7000 \mathrm{~V}$ and acceleration plate 2 at $6500 \mathrm{~V}$, and, initially, acceleration plate 3 was at $0 \mathrm{~V}$. A focusing voltage pulse of $740 \mathrm{~V}$ was then applied to acceleration plate 3 (ca. 2.1 $\mu s)$ after firing the desorbing laser for pulse focusing of the zinc mesoporphyrin parent ion. When PSPF was used (Figure 6b), the resolution of the molecular ion signal was increased to 2900 (f.t. /FWB), allowing clear distinction of the individual isotopic peaks in the molecular ion signal.

Figure 7 shows MALD/I spectra of angiotensin II (MW $1046 \mathrm{Da}$ ) in a 2,5-dihydroxybenzoic acid matrix both with and without PSPF taken at a 266-nm laser desorbing power of ca. $8 \mathrm{MW} / \mathrm{cm}^{2}$. The sample was prepared by codissolving the angiotensin II and 2,5-dihydroxybenzoic acid in a solution of ethanol (mole ratio 1:2000) and air-spraying the solution onto the probe tip, as previously described. The static acceleration electric field of $7000 \mathrm{~V} / \mathrm{cm}$ for the unfocused mass spectrum (Figure $7 \mathrm{a}$ ) was produced by using the same conditions as for the DLD/I mass spectrum in Figure 6a. The resolution of the angiotensin II molecular ion signal is ca. $\mathbf{5 0}$ (f.t./FWB) under these conditions. For the focused mass spectrum (Figure $7 \mathrm{~b}$ ), the initial accelerating electric fields were the same as for the DLD/I mass spectrum - in Figure $6 \mathrm{~b}$; however, a delay of ca. $2.5 \mu \mathrm{s}$ between the firing of the desorbing laser and application of the 871-V focusing voltage pulse was necessary to allow the larger mass angiotensin II parent ions to enter the pulse-focusing region. The PSPF technique allows the resolution of the molecular ion signal to be improved to 2750 (f.t./FWB) and further allows the distinction of a higher mass signal that has been tentatively identified as the sodiated $[M-H+23]$ ion. This higher mass ion signal clearly contributes to the low resolution in the static field TOF-MS mass spectrum.

Several important points relating to these initial experimental results warrant further discussion. First, although presented primarily for comparisons, it is clear that the resolution of the static electric field MALD/I signal is not as high as has been achieved by other researchers $[10,16,23]$. Both the weak source region electric field of just $7000 \mathrm{~V} / \mathrm{cm}$ and the nonoptimized method of sample preparation are likely causes of the lower resolution obscrved. At present, modifications of the linear TOF-MS instrumentation are in progress to allow higher source region acceleration electric fields to be used. In addition, improvements in sample preparation techniques are expected to yield higher ion signal resolutions for both the static field and PSPF mass spectra. Second, although PSPF yields
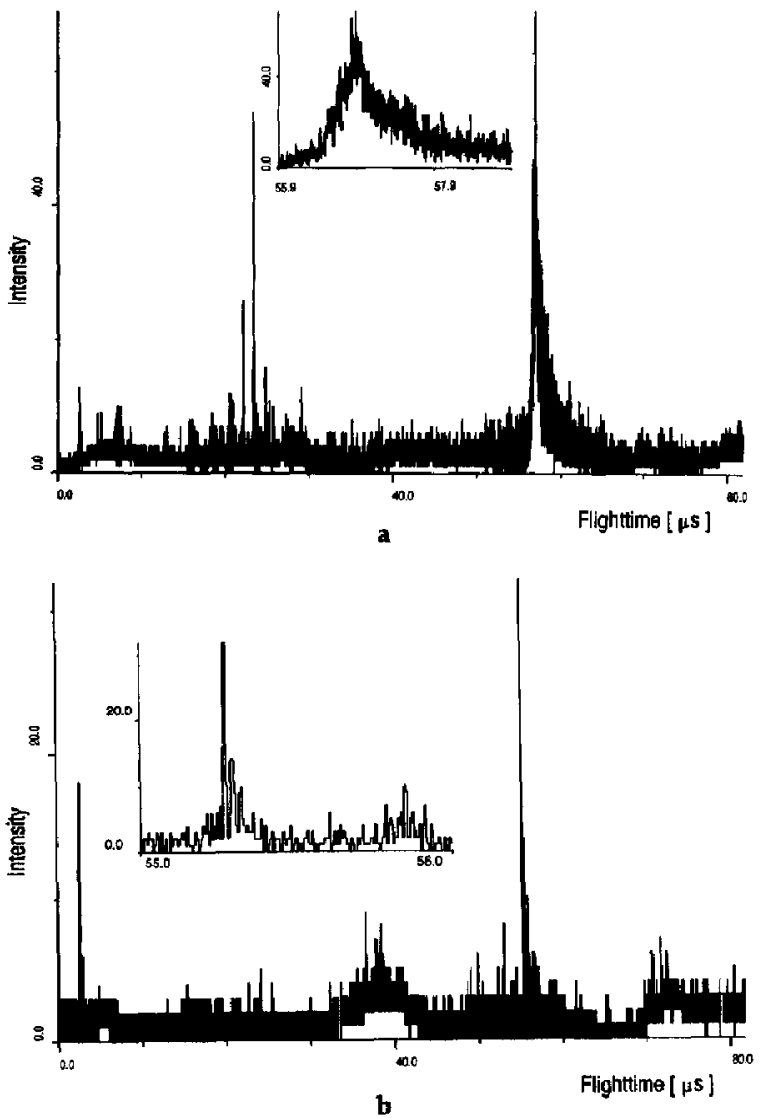

Figure 7. (a) MALD/I signal of angiotensin II in a 2,5-dihydroxybenzoic acid matrix taken under static acceleration electric field conditions; (b) MALD/I signal of angiotensin II in a 2,5-dihydroxybenzoic acid matrix taken with PSPF to enhance the resolution of the molecular ion. Insets show expansions of the molecular ion regions. Both spectra represent the sum of five laser shots.

significant improvement in ion signal resolution, a concurrent increase in the signal to noise is not observed. This is most likely due to less efficient ion collection at the reduced source region electric field used for PSPF of $500 \mathrm{~V} / \mathrm{cm}$. SIMION calculations have suggested a number of minor ion optic modifications that should allow the efficiency of the ion collection to be significantly enhanced at low source region electric fields. In addition, longer signal averaging can be used to improve the signal-to-noise ratio, although broadening in time of the detected ion signals by ca. $10 \mathrm{~ns}$ also results because of jitter in the pulsing and detection electronics. Third, it should be recognized that mass calibration using a simple linear expression relating ion flight times to the square root of the ion masses will no longer be appropriate for PSPF TOF-MS. At present, mass determination is achieved by calculating ion arrival times from first principles using the known instrumental conditions used to acquire a given mass 
spectrum. A more thorough discussion of this approach and a simplified expression for PSPF TOF-MS mass calibration will be presented in a future publication. Despite the present limitations, these initial results serve to clearly illustrate the improvements in mass resolution that may be achieved by using PSPF in combination with linear TOF-MS instrumentation.

\section{Conclusions}

The incorporation of PSPF in a linear TOF-MS offers a simple method for significantly improving the resolution of a large fraction of a recorded mass spectrum while maintaining good ion collection efficiencies and without degrading the resolution of the remaining mass spectrum. The PSPF TOF-MS technique has the capability to correct for both large distributions in initial ion translational energies and long durations of ion formation. These features may be particularly valuable for both DLD/I and MALD/I applications. Both the theoretical simulations and the initial experimental results presented in this report clearly demonstrate that PSPF in a linear TOF-MS is an effective method for improving the resolution of medium MW ion signals produced with both DLD/I and MALD/I techniques. Furthermore, it is anticipated that improvements beyond the mass resolution values achieved in these initial experiments will be realized through a number of relatively minor experimental modifications.

\section{Acknowledgments}

This work was supported by grants from Deutsche Forschungsgemeinschaft (CR917/1-2) and the Bundesministerium fuer Forschung und Technologie (13N5307-2). GRK gratefully acknowledges the fellowship support of the Alexander von Humboldt Stiftung, Bonn, Germany.

\section{References}

1. Honig, R. E.; Woolston, J. R. Appl. Phys. Lett. 1963, 2, 138.

2. For an early review see Conzemius, R. J.; Capellen, J. M. Int. J. Mass Spectrom. Ion Phys. 1980, 34, 197.

3. (a) Benninghoven, A., Ed. Springer Series in Chemical Physics 25: Ion Formation from Organic Solids; Springer-Verlag: Berlin, 1983; pp. 190-244; (b) Benninghoven, A., Ed. Springer Proceedings in Physics 9: Ion Formation from Oryanic Solias IFOS III; Springer-Verlag: Berlin, 1986; pp. 142-162.

4. Van Vaeck, L.; Bennett, J.; Van Epsen, P.; Schweikert, E.; Gijbels, R.; Adams, F.; Lauwers, W. Org. Mass Spectrom. 1989, 24. 797 .

5. Branan, D. M.; Hoffman, N. W.; McElroy, E. A.; Ramage, D. L.; Robbins, M. J.; Eyler, J. R.; Watson, C. H.; deFur, P.; Leary, J. A. Inorg. Chem. 1990, 29, 1915.

6. Karas, M.; Bachmann, D; Bahr, U; Hillenkamp, F. Int. J. Mass Spectrom. lon Processes 1987, 78, 53.

7. Tanaka, K.; Waki, H.; Ido, Y.; Anita, S.; Yoshida, Y.; Yoshida, T. Rapid Commun. Mass Spectrom. 1988, 2, 151.

8. Karas, M.; Bahr, U.; Ingendoh, A.; Hillenkamp. F. Angew. Chem. Int. Ed. Engl. 1989, 28, 760.

9. (a) Karas, M.; Hillenkamp, F. Anal. Chem. 1988, 60, 2301; (b)
Overberg, A.; Karas, M.; Hillenkamp, F. Rapid Commun. Mass Spectrom. 1991, 5, 128; (c) Overberg, A.; Karas, M.; Bahr, U.; Kaufmann, R.; Hillenkamp, F. Rapid Commun. Mass Spectrom. 1990, 4, 293.

10. (a) Beavis, R. C.; Chait, B. Rapid Commun. Mass. Spectrom. 1989, 3, 233; (b) Beavis, R. C.; Chait, B. Rapid Commun. Mass Spectrom. 1989, 3, 432; (c) Beavis, R. C; Chait, B. Rapid Commun. Mass Spectrom. 1989, 3, 436; (d) Beavis, R. C.; Chait, B. T. Anal. Chem. 1990, 62, 1836.

11. (a) Spengler, B.; Cotter, R. J. Anal. Chem. 1990, 62, 793; (b) Spengler, B.; Pan, Y.; Cotter, R. J.; Kan, L. Rapid Commun. Mass Spectrom. 1990, 4, 99.

12. (a) Salchpour, M.; Perera, I.; Kjellberg, J.; Hedin, A.; Islamian, M. A.; Hakansson, P.; Sundqvist, B. U. R. Rapid Commun. Mass Spectrom. 1989, 3, 259; (b) Perera, I. K.; Uzcategui, E; Hakansson, P.; Brinklmalm, G.; Pettersson, G.; Johansson, G.; Sundqvist, B. U. R. Rapid Commun. Mass Spectrom. 1990, 4, 285.

13. (a) Nelson, R. W.; Thomas, R. M.; Williams, P. Rapid Commun. Mass Spectrom. 1990, 4, 301; (b) Nelson, R; Rainbow, M.; Lohr, D,; Williams, P. Science, 1989, 246, 1585.

14. (a) Huang, L. Q.; Conzemius, R. J.; Junk, G. A.; Houk, R. S. Anal. Chem. 1988, 60, 1490; (b) Denoyer, E,; Van Grieken, R.; Adams, F.; Natusch, D. Anal. Chem. 1982, 54, 26A.

15. (a) Karas, M.; Bahr, U.; Ingendoh, A.; Hillenkamp, F. Angew. Chem. Int. Ed. Eng. 1989, 28, 760; (b) Kinsel, G. R.; Lindner, J.; Koester, C,; Zipperer, K.; Grotemeyer, J. Inst. Phys. Conf. Ser. 1990, 114, 155.

16. Beavis, R.; Chait, B. Rapid Commun. Mass Spectrom. 1989, 3, 233.

17. Spengler, B.; Kirsch, D.; Kaufmann, R. Rapid Commun. Mass Spectrom. 1991, 5, 198.

18. (a) Karas, M.; Bahr, U,; Hassenbuerger, A.; Ingendoh, A.; Overberg, A.; Stahl, B; Strupat, K.; Hillenkamp, F. Proceedings of the 39 th ASMS Conference on Mass Spectrometry and Allied Topics; Nashville, TN, May 1991, p. 356; (b) Holle, A.; Mayer, F.; Schaefer, R.; Frey, R. Proceedings of the 39th ASMS Conference on Mass Spectrometry and Allied Topics; Nashville, TN, 1991, p. 936.

19. (a) Price, D.; Milnes, G. J. Int. I. Mass Spectrom. Ion Proc. 1990, 99, 1; (b) Campana, J. E. Anal. Instrumen. 1987, 16, 1; (c) Le Beyec, Y. Adv. Mass Spectron. 1989, 11, 126; (d) Boesl, U.; Weinkauf, R.; Schlag, E. W. Int. I. Mass Spectrom. Ion Proc. 1991, 112, 121.

20. Yang, M.; Reilly, J. P. Int. J. Mass Spectrom. Ion Proc. 1987, 75, 209.

21. (a) Van der Peyl, G. J. Q.; Van der Zande, W. I.; Kistemaker, P. G. Int. J. Mass Spectrom. Ion Phys. 1984, 62, 51; (b) Antonov, V. S.; Letokhov, V. S.; Shibanov, A. N. Appl. Phys. 1981, 25. 71; (c) Stoll, R.; Roellgen, F. W. Z. Naturforsch. A 1982, 37, 9.

22. Mauney, T.; Adams, F. Int. J. Mass Spectrom. Ion Proc. 1984, $59,103$.

23. (a) Ens, W.; Mao, Y.; Mayer, F, Standing, K. G. Rapid Commun. Mass Spectrom. 1991, 5, 117; (b) Beavis, R. C.; Chait, B. T. Chem. Phys. Lett. 1991, 181, 479.

24. Beavis, R. C.; Chait, B. T. In Ion Formation from Organic Solids (IFOS V), Proceedings of the 5th International Conference; Hedin, A.; Sundqvist, B. U. R.; Benninghoven, A., Eds.; Wiley: Chichester (England), 1990; pp. 125-130.

25. (a) Mamyrin, B. A.; Karataev, V. I.; Shmikk, D. V; Zagulin, V. A. Sov. Phys. JETP 1973, 37, 45; (b) Karataev, V. I.; Mamyrin, B. A.; Shmikk, D. V. Sov. Phys. Tech. Phys. 1972, 16, 1177; (c) Mamyrin, B. A.; Shmikk, D. V. Sov. Phys. JETP 1979, 49,762 .

26. Gohl, W.; Kutscher, R.; Laue, H. J.; Wollrik, H. Int. J. Mass Spectrom. Ion. Phys. 1983, 48, 411.

27. (a) Boesl, U.; Neusser, H. J.; Weinkauf, R.; Schlag, E. W. I. 
Phys. Chem. 1982, 86, 4857; (b) Kuehlewind, J.; Neusser, H. J.; Schlag, E. W. Int. J. Mass Spectrom. Ion Phys. 1983, 51, 255; (c) Boesl, U.; Grotemeyer, J.; Walter, K; Schlag, E. W. Anal. Instrum. 1987, 16, 151.

28. (a) Wiley, W. C.; McLaren, I. H. Rev. Sci. Instrum. 1955, 26, 1150; (b) Erickson, E. D.; Yefchak, G. E.; Enke, C. G.; Holland, J. F. Int. J. Mass Spectrom. Ion Proc. 1990, 97, 87.

29. (a) Muga, M. L. Anal. Instrum. 1987, 16, 31; (b) Muga, M. L. U.S. Patent 4,458,149, July 3, 1984 .
30. (a) Marable, N. L.; Sanzone, G. Int. J. Mass Spectrom. Ion Phys. 1974, 13, 185; (b) Browder, J. A.; Miller, R. L.; Thomas, W. A.; Sanzone, G. Int. J. Mass Spectrum. Ion Phys. 1981, 37, 99.

31. Yefchak, G. E.; Enke, C. G.; Holland, J. F. Int. J. Mass Spectrom. Ion Proc. 1989, 87, 313.

32. Kinsel, G. R.; Johnston, M. V. Int. J. Mass Spectrom. Ion Proc. 1989, 91, 157.

33. Kinsel, G. R.; Mowry, C. D.; McKeown, P. J.; Johnston, M. V. Int. J. Mass Spectrom. Ion Proc. 1991, 104, 35. 